Check for updates

Cite this: DOI: 10.1039/c7ta08972d
Received 12th October 2017

Accepted 29th November 2017

DOI: $10.1039 / c 7 t a 08972 d$

rsc.li/materials-a

\section{A facile nanocomposite strategy to fabricate a rGO-MWCNT photothermal layer for efficient water evaporation $\uparrow$}

\author{
Yuchao Wang, (D) a Canzhu Wang, ${ }^{\text {ab }}$ Xiangju Song, ${ }^{\text {ac }}$ Suresh Kumar Megarajan $\mathbb{D}^{a}$ \\ and Heqing Jiang (iD *a
}

\begin{abstract}
Solar-driven water evaporation assisted by photothermal membranes is considered as one of the sustainable and cost-effective strategies for pure water generation and wastewater treatment. Herein, we report a facile but effective approach to improve the photothermal performance by combining $2 \mathrm{D}$ reduced graphene oxide ( $\mathrm{rGO}$ ) and $1 \mathrm{D}$ multi-walled carbon nanotubes (MWCNTs), which have different nanomorphologies. The photothermal layer can be easily deposited on different substrate materials via simple vacuum assistance. Such a composite photothermal layer shows a rough surface with a controllable nano-structure, which can thus optimize solar light harvesting. On the other hand, the formation of a loose internal porous structure and suitable wettability ensure water transport inside the photothermal layer during evaporation. The surface temperature reaches as high as $78{ }^{\circ} \mathrm{C}$ even under one sun irradiation $\left(1 \mathrm{~kW} \mathrm{~m}{ }^{-2}\right)$, which is $10^{\circ} \mathrm{C}$ higher than the result of pure $\mathrm{rGO}$ membranes. When loaded on a PVDF substrate, the rGO-MWCNT based membrane is flexible and shows an obvious improvement in the evaporation rate, about $79.0 \%$ and $8.9 \%$ higher than those of pure rGO and MWCNT membranes, respectively. The solar thermal conversion efficiency can reach up to $80.4 \%$ without any extra accessory for thermal management. Based on our results, the nanocomposite strategy is facile and effective for the development of novel photothermal membranes for high-efficiency evaporation, and contributes to the widespread application in the fields of desalination and wastewater treatment.
\end{abstract}

\section{Introduction}

Clean water scarcity is an urgent global issue restricting sustainable development with increasing demand from the rapidly growing population and water pollution., ${ }^{1,2}$ Among a range of ever-evolving technologies, solar-driven water evaporation is considered to be one of the ideal ways to obtain fresh water with minimum greenhouse gas emissions. ${ }^{3,4}$ During the history of mankind, solar-driven evaporation has been emerging as one of the most promising solar energy technologies, because of its potential applications in the fields of desalination, ${ }^{5,6}$ water purification, ${ }^{7}$ and liquid-phase separation..$^{\mathbf{8 , 9}}$ The advantage of the solar distillation process lies in the direct use of solar irradiation as the energy source, which is inexhaustible and widely distributed, and therefore beneficial for

\footnotetext{
${ }^{a}$ Qingdao Key Laboratory of Functional Membrane Material and Membrane Technology, Qingdao Institute of Bioenergy and Bioprocess Technology, Chinese Academy of Sciences, Qingdao 266101, China.E-mail: jianghq@qibebt.ac.cn

${ }^{b}$ Institute of Materials Science and Engineering, Ocean University of China, Qingdao 266101, China

'University of Chinese Academy of Sciences, Beijing 100049, China

$\dagger$ Electronic supplementary information (ESI) available: SEM images, membrane characterization and performance results. See DOI: 10.1039/c7ta08972d
}

making portable desalination kits for decentralized water supply systems. ${ }^{\mathbf{1 0 , 1 1}}$ However, traditional solar steam generation processes suffer from poor efficiency due to the nature of water. Water, as an extremely poor light absorber, is difficult to use for harvesting light within almost the entire solar spectrum. ${ }^{\mathbf{1 2}}$ Based on our previous studies, ${ }^{\mathbf{1 2}, 13}$ the efficiency of solar-driven water evaporation depends strongly on the assistance of photothermal materials that are beneficial to exclusively heat up the interfacial water and decrease the heat transfer to the nonevaporated portion of the bulk water.

Utilizing rationally designed photothermal materials to localize the light absorption in the top water surface has recently been emerging as an appropriate approach to accelerate the water evaporation. From the studies reported in recent years, ${ }^{\mathbf{1 4}}$ it becomes apparent that ideal photothermal materials for water evaporation should integrate several properties including (i) high solar absorption in the entire solar spectrum range, (ii) efficient light-to-heat conversion without energy loss, and (iii) a porous structure with a wettable surface for feasible water transport from a bulk water body to the evaporation interface. So far for photothermal materials, noble metal nanoparticles, ${ }^{15,16}$ composite particles, ${ }^{17}$ polypyrrole coating, ${ }^{6}$ carbon materials ${ }^{\mathbf{1 8 - 2 0}}$ and plasmonic absorbers ${ }^{21}$ have been investigated and found to exhibit much faster evaporation of 
water steam compared to pure water without any photothermal materials under irradiation. ${ }^{22}$ Among them, graphene is a suitable and practical candidate for photothermal water evaporation because of its low specific heat, high broadband solar harvesting, stable physical and chemical properties, controllable morphology and low cost. ${ }^{23-25}$

Graphene, a two-dimensional (2D) nanomaterial, can be easily processed into a membrane on porous substrates by vacuum filtration. ${ }^{26,27}$ However, the as-prepared graphene membrane usually shows a smooth surface with weak light absorption because of its strong light reflection. Besides that, the membrane is constructed by densely stacked graphene sheets, and it is difficult for water molecules to pass through them without an external driving force. ${ }^{28-30}$ To overcome these problems, graphene aerogels with a rough surface and porous structure have been developed by several groups. ${ }^{31-33}$ However, these syntheses need special techniques (vacuum freeze-drying) or extra treatment processes (template removal). And normal aerogels usually suffer from low mechanical stability, causing limitations in large scale applications. ${ }^{34}$ Therefore, it is still a challenge to develop an easy-to-handle and general strategy to fabricate a stable and economical graphene based photothermal membrane with controllable surface roughness and a suitable porous structure on various substrates for high evaporation performance.

In this work, we report a nanocomposite strategy to fabricate a carbon-based photothermal layer by combining reduced graphene oxide (rGO) and multi-walled carbon nanotubes (MWCNTs) for high-efficiency solar-driven water evaporation. With rational design, the surface roughness of the rGOMWCNT photothermal layer can be controlled and optimized to enhance the solar irradiation absorption in a wide wavelength range by minimizing light reflection. Meanwhile, the gaps formed by MWCNT doping inside the photothermal layer improve the water transport through the capillary effect. Such a carbon composite can be deposited and form a stable photothermal coating easily on several kinds of polymer or inorganic substrates, including polyvinylidene fluoride (PVDF) membranes, anodic aluminum oxide (AAO), polysulfone (PSf) membranes, air-laid paper and phenolic resin (RF) sponge. With a mixture of MWCNTs, the photothermal evaporation rate of the rGO-MWCNT composite membrane is remarkably enhanced over $79.4 \%$ and $8.9 \%$ compared to pure rGO and MWCNTs, respectively. The solar thermal conversion efficiency can reach up to $80 \%$ with an optimized composite ratio under one sun irradiation. This nanocomposite concept to rationally design carbon-based photothermal membranes is a promising attempt to develop a cost-effective and general approach to prepare photothermal membranes for high-efficiency evaporation, which will be beneficial for a wide range of applications in the fields of desalination, sewage treatment, etc.

\section{Experimental}

\subsection{Reagents and materials}

A hydrophilic polyvinylidene fluoride (PVDF) membrane with a stated pore size of $0.22 \mu \mathrm{m}$ was purchased from Millipore. An anodic aluminum oxide (AAO) membrane with a pore size of $0.20 \mu \mathrm{m}$ was purchased from Whatman. Polysulfone (PSf) membrane was purchased from Hangzhou Water Treatment Center. Multi-walled carbon nanotubes (MWCNTs, O.D. $\times$ L 20$40 \mathrm{~nm} \times 10-30 \mu \mathrm{m})$ were bought from Aladdin Chemical Co., Ltd. Graphite powder $(\leq 30 \mu \mathrm{m})$, sulfuric acid $\left(\mathrm{H}_{2} \mathrm{SO}_{4}\right)$, phosphoric acid $\left(\mathrm{H}_{3} \mathrm{PO}_{4}\right)$, potassium permanganate $\left(\mathrm{KMnO}_{4}\right)$, hydrogen peroxide solution $\left(\mathrm{H}_{2} \mathrm{O}_{2}\right)$, hydrochloric acid $(\mathrm{HCl})$, nitric acid $\left(\mathrm{HNO}_{3}\right)$, sodium chloride $(\mathrm{NaCl})$, sodium hydroxide $(\mathrm{NaOH})$ and hydroiodic acid (HI) were purchased from Sinopharm Chemical Reagent Co., Ltd. Air-laid paper (Jet-Spun cloth) was obtained from DuPont. Phenolic resin foam, a kind of water absorption sponge, was bought from Yixuan. The phenolic resin sponge was cut into pieces $32 \mathrm{~mm}$ in diameter and $1 \mathrm{~mm}$ in thickness. All the reagents were of analytical grade and used as received without further purification. De-ionized water was used in all the experiments.

\subsection{Preparation of carbon-based photothermal membranes}

Graphene oxide (GO) was synthesized based on an improved Hummers' method. ${ }^{35}$ Well-dispersed MWCNTs were obtained by a typical acid oxidation procedure. More details are shown in the ESI. $\dagger$

$4 \mathrm{~mL}$ of suspension with different ratios of GO to acid-treated MWCNTs was mixed uniformly by ultrasonication for $30 \mathrm{~min}$. Then the suspension was loaded on the PVDF membrane to form the carbon-based photothermal layer with the assistance of vacuum. The obtained membranes were dried at $80{ }^{\circ} \mathrm{C}$ overnight and reduced with $\mathrm{HI}$ vapor in a sealed container at $90{ }^{\circ} \mathrm{C}$ for $2 \mathrm{~h}$. Reduced graphene oxide (rGO) was generated during the reduction reaction. To remove the residual iodine, ethanol and water were used to wash the membranes alternately. After drying completely, the resultant membranes before and after reduction were denoted as $\mathrm{GO}-x \mathrm{C}$ and $\mathrm{G}-x \mathrm{C}$, respectively, with $x$ being the percentage of MWCNTs.

\subsection{Characterization}

Scanning electron microscopy (SEM) images were taken with a Hitachi S-4800. The diffuse reflectance and transmittance UVvis-NIR absorption spectra were recorded on a spectrophotometer (Hitachi U-4100). The surface morphology and roughness were measured using an Atomic Force Microscope (AFM, Agilent 5400). The elements were analyzed using X-ray photoelectron spectroscopy (XPS, Shimadzu KROTAS AMICUS spectrometer). Contact angles were measured on a commercial contact angle system (OCA 25 of Data-Physics) at ambient temperature using a $3 \mu \mathrm{L}$ droplet as the indicator. FTIR spectra were collected on a Nicolet iS10 FTIR spectrometer. The temperature and IR images were captured using an FLIR One thermal imager connected to an iphone 6.

\subsection{Solar evaporation performance measurement}

Water was placed in a glass container with a mouth diameter of $40 \mathrm{~mm}$. Simulated solar irradiation was provided by a xenon lamp (CEL-PE300L-3A, CEAULIGHT, Beijing, China), and the light intensity was kept at $1 \mathrm{~kW} \mathrm{~m}^{-2}$ (one sun). A polystyrene 
(PS) ring with an inner diameter of $26 \mathrm{~mm}$ and an outer diameter of $40 \mathrm{~mm}$ was covered on the as-prepared membrane sample as a mask. The tested samples applied in photothermal evaporation were kept in a circular area with a diameter of $26 \mathrm{~mm}$. The membrane sample was floatable and placed on top of the water in the glass container, and simulated solar light was shone onto the material vertically from the top. The glass container was placed on an electronic analytical balance, which was connected to a computer for real-time monitoring of water loss due to evaporation. All the test data were obtained at an ambient temperature of $24 \pm 0.5{ }^{\circ} \mathrm{C}$ and a relative humidity of $50 \%$. The experimental value of the water evaporation rate $\left(v_{\mathrm{e}}\right)$ was calculated using eqn (1): ${ }^{12}$

$$
v_{\mathrm{e}}=\frac{\mathrm{d} m}{S \times \mathrm{d} t}
$$

where $m$ is the mass of the evaporated water, $S$ is the surface area of the photothermal membrane (circular area with a diameter of $26 \mathrm{~mm}$ ), and $t$ is the time.

\section{Results and discussion}

\subsection{Fabrication of the rGO-MWCNT photothermal membrane}

rGO-MWCNT composite photothermal membranes were prepared as shown in the schematic in Fig. 1. Briefly, a certain amount of graphene oxide (GO) and acid-treated multi-walled carbon nanotube (MWCNT) aqueous dispersion mixture was deposited onto the commercial hydrophilic polyvinylidene fluoride (PVDF) membrane by vacuum filtration. More porous substrates, including anodic aluminum oxide (AAO), polysulfone (PSf) membrane, air-laid paper and phenolic resin (RF) sponge, were also used for GO-MWCNT deposition. After the reduction of $\mathrm{GO}$ to $\mathrm{rGO}$ by $\mathrm{HI}$, a stable and flexible photothermal membrane was formed with reduced graphene oxide (rGO) sheets and MWCNTs. The residual iodine can be easily removed with ethanol and water.

During the vacuum filtration, the GO sheets tend to stack closely together because of their flat $2 \mathrm{D}$ feature. ${ }^{29}$ After reduction, a mirror-like surface of rGO membrane with few surface details at the microscale is usually built by rGO itself (Fig. S1 in the ESI $\dagger$ ). Fig. 2 and $\mathrm{S} 2 \dagger$ show the SEM images of the asprepared carbon-based photothermal layers on PVDF. Only few wrinkles can be observed on the surface of the pure rGO membrane on the PVDF substrate from its SEM image (Fig. 2a). After MWCNTs were added into the system, the composite photothermal membranes exhibited more obvious wrinkles. As shown in the SEM image of the G-88C sample with $88 \mathrm{wt} \%$ MWCNTs, the mountain-like structures were distributed all over the surface of the membrane and enhanced the roughness. However, further increasing the percentage of MWCNTs over $94 \%$ would cause fewer surface details, similar to that observed for sample G-94C (Fig. 2c). In comparison, the MWCNT layer without rGO shows a generally smooth surface (Fig. 2d). To quantitatively evaluate the difference between the surface morphology of these samples, AFM was employed to examine their surface roughness $\left(S_{\mathrm{q}}\right)$ (Fig. 2e-h). Compared with the pure rGO membrane, sample G-88C shows a higher $S_{\mathrm{q}}$ up to $0.212 \mu \mathrm{m}$ indicating a rougher top surface, while the $S_{\mathrm{q}}$ of sample G-94C reduces to $0.158 \mu \mathrm{m}$, which is similar to the pure rGO's value of $0.153 \mu \mathrm{m}$. The observed SEM images are therefore clearly substantiated by AFM results. From SEM and AFM results, we can therefore clearly infer that the MWCNTs with 1D morphology would disturb the ordered arrangement of 2D rGO nanosheets and lead to an increase in surface roughness. However, when the amount of rGO is too low, the MWCNTs tend to form a uniform layer on the substrate by themselves with a smooth surface.
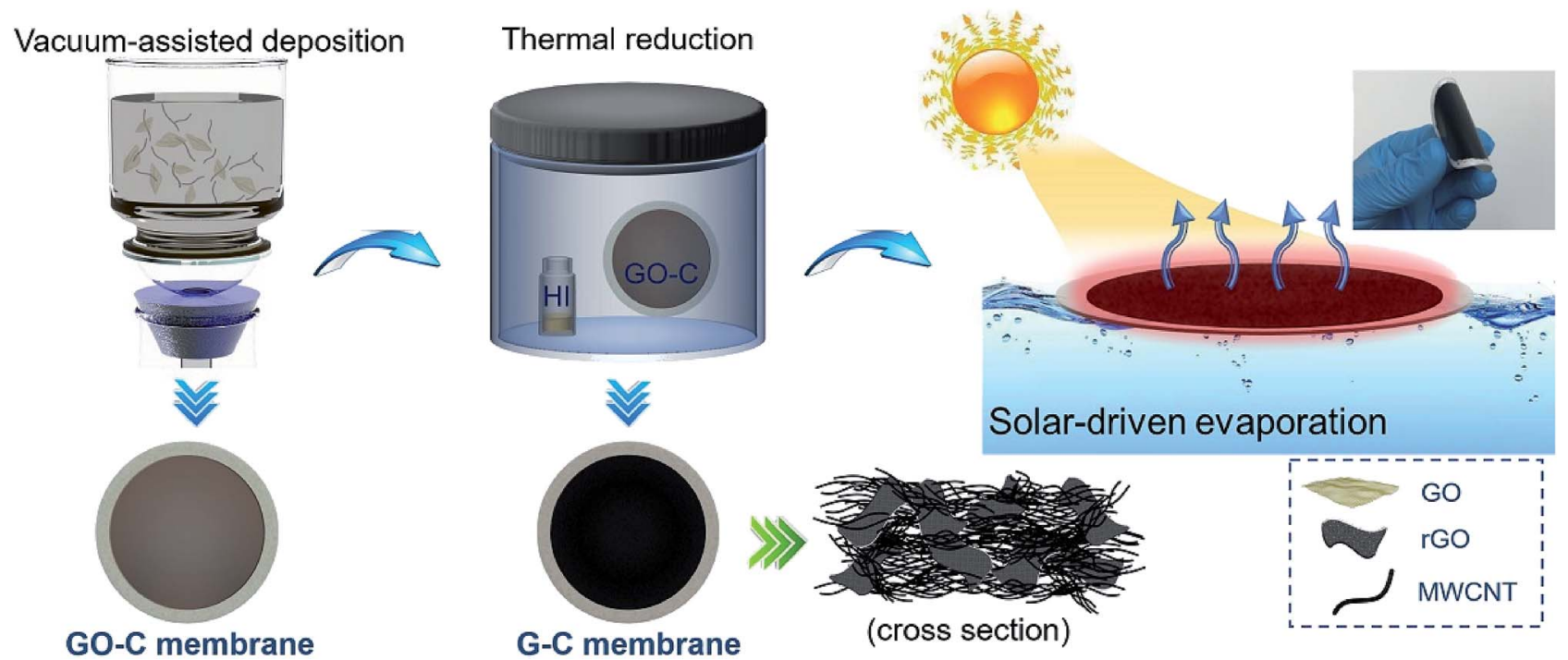

Fig. 1 Schematic illustration of the preparation of the rGO-MWCNT photothermal layer on a porous substrate. GO and acid-treated MWCNTs were deposited on the porous substrate under vacuum. After thermal reduction by $\mathrm{HI}$, the obtained $\mathrm{rGO}-\mathrm{MWCNT}$ membrane showed high performance for photothermal evaporation. The inset is a digital image of G-88C. 

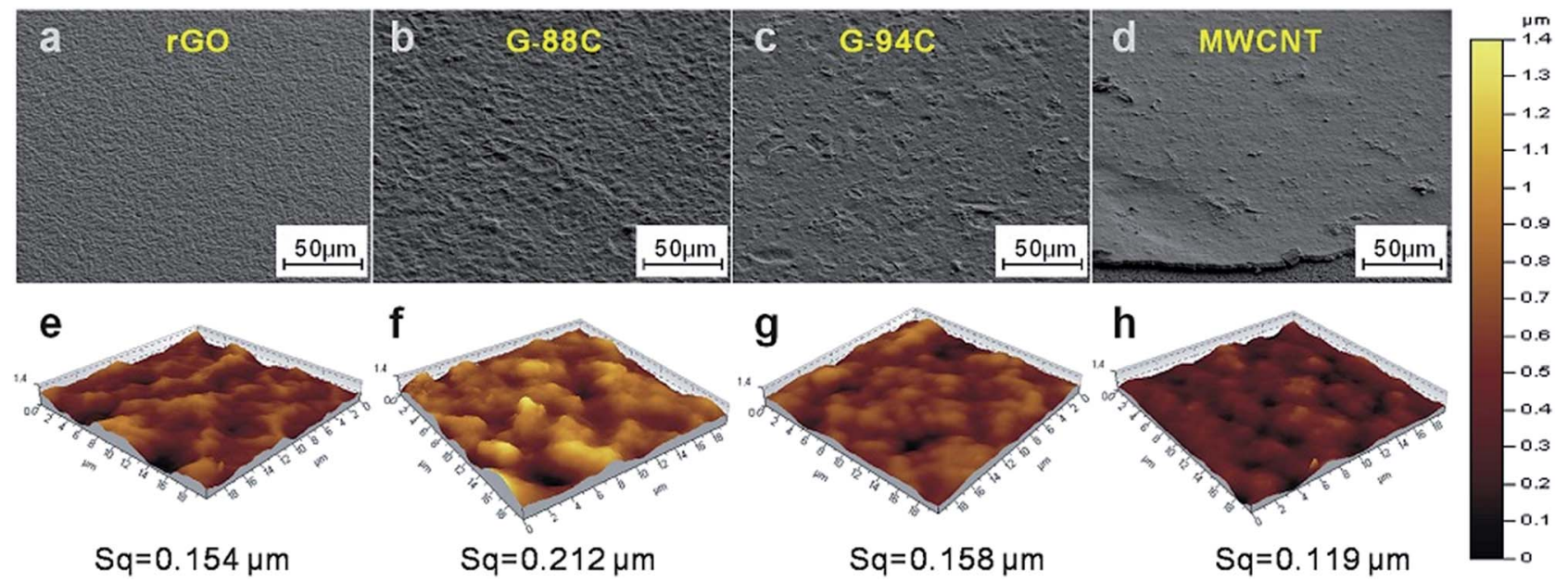

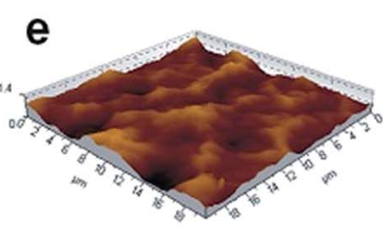

$S q=0.154 \mu \mathrm{m}$

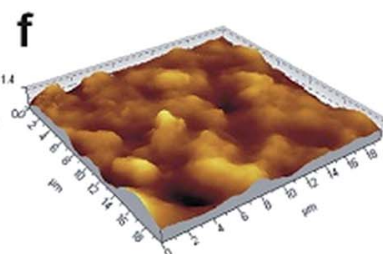

$\mathrm{Sq}=0.212 \mu \mathrm{m}$

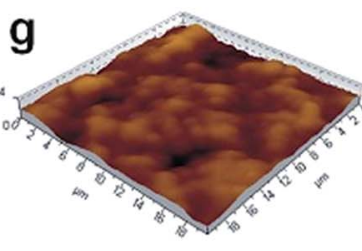

$\mathrm{Sq}=0.158 \mu \mathrm{m}$

$S q=0.119 \mu \mathrm{m}$

Fig. 2 Tilt view SEM images and the corresponding AFM images of the top surface of the rGO layer (a and e), rGO-MWCNT combined layer with MWCNT ratios of $88 \%$ (b and f) and 94\% (c and g), and pure MWCNT layer ( $d$ and h), on top of commercial PVDF substrates.

\subsection{Effect of rGO to MWCNT ratio on light harvesting}

The light reflection and transmission properties of the photothermal membranes were carefully investigated to evaluate the solar light-harvesting efficiency (Fig. $3 \mathrm{a}$ and b). In the wavelength range of $300-2500 \mathrm{~nm}$, all samples showed quite low transmission close to zero. This indicates that the light in a wide wavelength range has been totally hindered by the photothermal layer. The diffuse reflection of the pure rGO layer on PVDF is about $13.3 \%$, which is relatively high, because of the smooth surface, while the diffuse reflections of rGO-MWCNT composite layers decrease from 10.2 to $4.7 \%$ with an increase of the MWCNT amount from 75 to $88 \mathrm{wt} \%$. Such low light reflection of the photothermal layers is attributed to the great light absorption of the carbon nanotubes as well as the additional surface micro-structure (Fig. 3c). The small diffuse reflectivity and almost zero transmission indicate nearly full light harvesting of the rGO-MWCNT membrane.

The improved solar light harvesting due to the rationally designed surface micro-structure can also be confirmed by surface temperature measurement under one sun light irradiation, which is widely used to evaluate the light-to-heat conversion performance of membranes. The equilibrium surface temperatures of the as-prepared samples were recorded from the IR images in Fig. 3d-h. The rGO layer on PVDF showed an equilibrium temperature of around $66^{\circ} \mathrm{C}$ under $1 \mathrm{~kW} \mathrm{~m}{ }^{-2}$
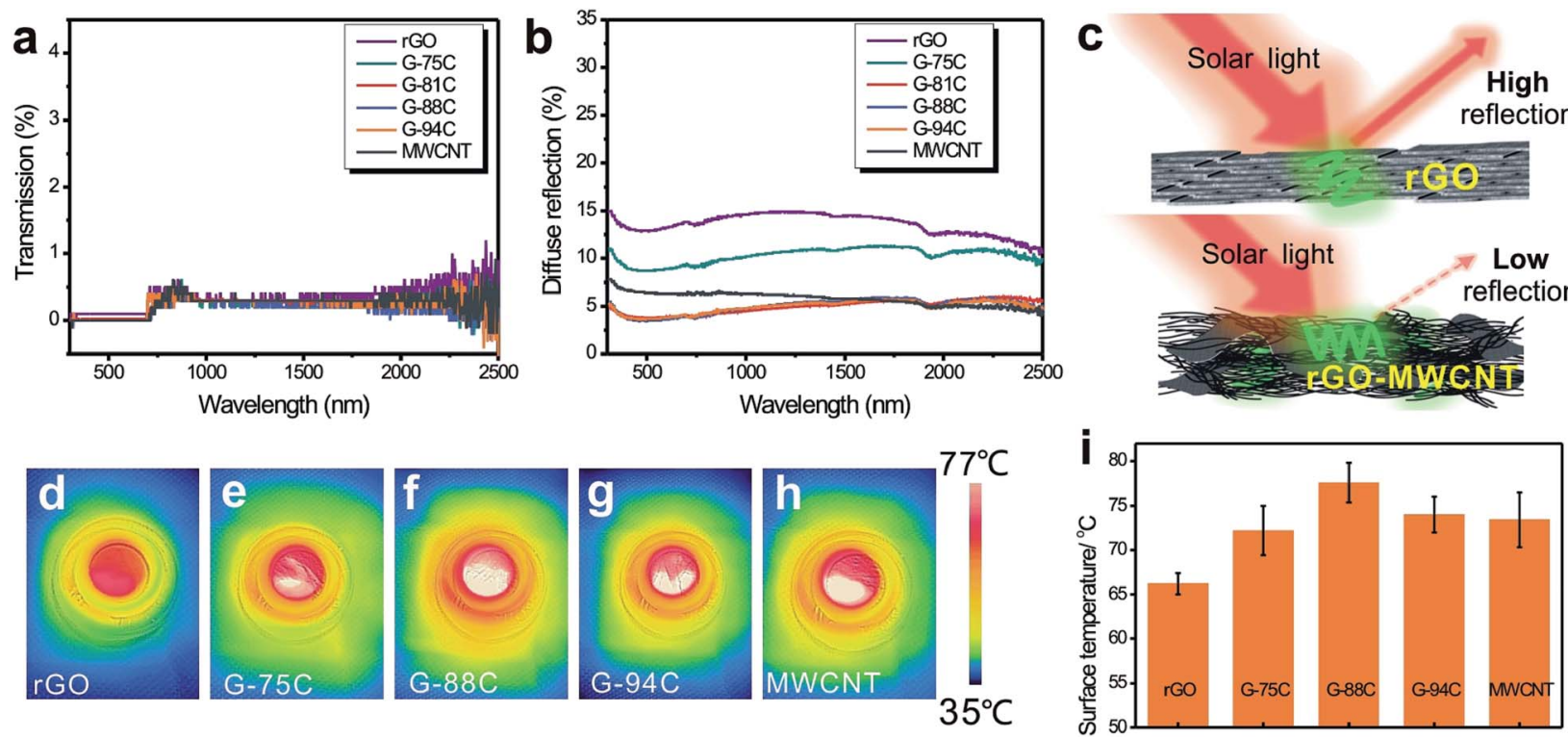

Fig. 3 UV-vis-NIR transmission (a) and diffuse reflection (b) spectra of the carbon-based photothermal layers. Schematic illustration of the photothermal layer to decrease the light reflection (c). The IR images of carbon-based photothermal layers on PVDF under simulated solar light irradiation for $30 \mathrm{~min}$ in air at room temperature $(\mathrm{d}-\mathrm{h})$, and the equilibrium temperatures of carbon-based photothermal layers on PVDF substrates which were recorded from the IR images (i). 
light irradiation for $30 \mathrm{~min}$ at room temperature (RT, $24{ }^{\circ} \mathrm{C}$ ), while the rGO-MWCNT photothermal layers showed a gradual temperature rise with the increase of MWCNT weight percentage. The surface temperature of sample $\mathrm{G}-88 \mathrm{C}$ reached up to $77.6 \pm 2.2{ }^{\circ} \mathrm{C}$. This is over $10{ }^{\circ} \mathrm{C}$ higher than the result of the pure rGO sample. This surface temperature rise can be ascribed to the better light-harvesting efficiency caused by the higher surface roughness. Having confirmed that the rGOMWCNT layer obtained following the nanocomposite strategy has a significant enhancement of photothermal performance on the commercial PVDF substrate, we subsequently tested the surface temperatures of the carbon-based photothermal layers on other inorganic and organic porous substrates, including anodic aluminum oxide (AAO), polysulfone (PSf) membrane, air-laid paper and phenolic resin (RF) sponge. The G-88C samples on all substrates exhibited higher surface equilibrium temperatures than the samples with a single component (Fig. 3i and S3†), indicating that the nanocomposite strategy is a general strategy for the deposition of high efficiency photothermal materials on different substrates. In general, one of the main uses of MWCNTs in combination with rGO is to form a rougher surface to minimize the diffuse reflection for high light harvesting during the photothermal process.

\subsection{Effect of rGO-MWCNT composition on water transport}

Generally, the water transfer behavior from the bulk part to the evaporation interface is also an important factor for the water evaporation rate. Another objective of introducing MWCNTs into the rGO membrane is to improve the porosity of the membrane to get water transfer channels and thus ensure a good water transport behavior.

Fig. 4 presents the cross-sectional views of the carbon-based layers on PVDF. The pure rGO sample shows a clear lamellar structure due to the dense stack of nanosheets with a thickness around $0.67 \mu \mathrm{m}$. However, the thicknesses of rGO-MWCNT photothermal layers on PVDF increase to around 1.37-1.67 $\mu \mathrm{m}$. As shown in Fig. 4b, the thicker composite layer indicates a loose structure of the G-88C photothermal membrane, under

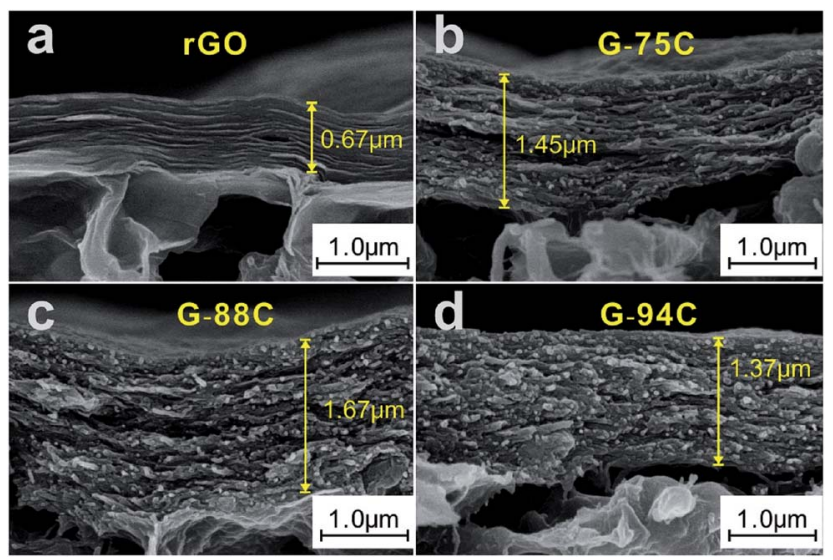

Fig. 4 Cross-sectional SEM images of rGO (a) and rGO-MWCNTs with different MWCNT ratios $(b-d)$ on top of PVDF membranes. a fixed total carbon amount. With the different nanomorphologies, MWCNTs intercalate the interlaminated rGO nanosheets, resulting in a loose structure in rGO-MWCNT photothermal layers. This loose structure is expected to be beneficial for water transport during evaporation. ${ }^{13}$ With more MWCNTs, G-94C shows a similar internal structure consisting of nanotubes with the pure MWCNT membrane (Fig. S4 $\dagger$ ). It is known that the pure rGO membrane easily cracks and separates from the substrate during bending. ${ }^{26}$ MWCNTs with 1D morphology are able to fix the rGO nanosheets and avoid cracks. As shown in Fig. S5, $\dagger$ the flexible rGO-MWCNT photothermal membranes are stable without cracks even after repeated bending.

Wettability is an important factor that influences photothermal evaporation, since it can directly affect the water transport behavior..$^{32,36-38}$ The water contact angles (CA) of carbon-based layers were characterized to analyze the wetting properties (Fig. 5a and $56 \dagger$ ). Because the water droplet would spread out gradually, all CA data were collected as soon as the droplets touched the carbon-based layer surfaces. The rGO membrane showed a weak hydrophilic behavior with a larger contact angle of $83 \pm 4^{\circ}$ after HI reduction. However, the MWCNT composition significantly changed the wettability of the photothermal layer. The G-88C sample has a smaller contact angle of $68 \pm 3^{\circ}$, exhibiting better hydrophilic features and dispersing the water droplet quickly. Similar wetting results were also found for the pure MWCNT layer. With the loose structure of rGO-MWCNT membranes, the better wettability would ensure smooth water transport during evaporation.

FTIR was used to detect the functional groups on the surface of carbon-based photothermal layers during the reduction process (Fig. $5 \mathrm{~b}$ and $\mathrm{S7} \dagger$ ). Several intense bands at $3360 \mathrm{~cm}^{-1}$, $1725 \mathrm{~cm}^{-1}$ and $1632 \mathrm{~cm}^{-1}$ were recorded in the FTIR spectrum, representing hydroxyl, carbonyl and carboxyl groups respectively. ${ }^{39}$ After HI treatment, these bands of oxygen-containing
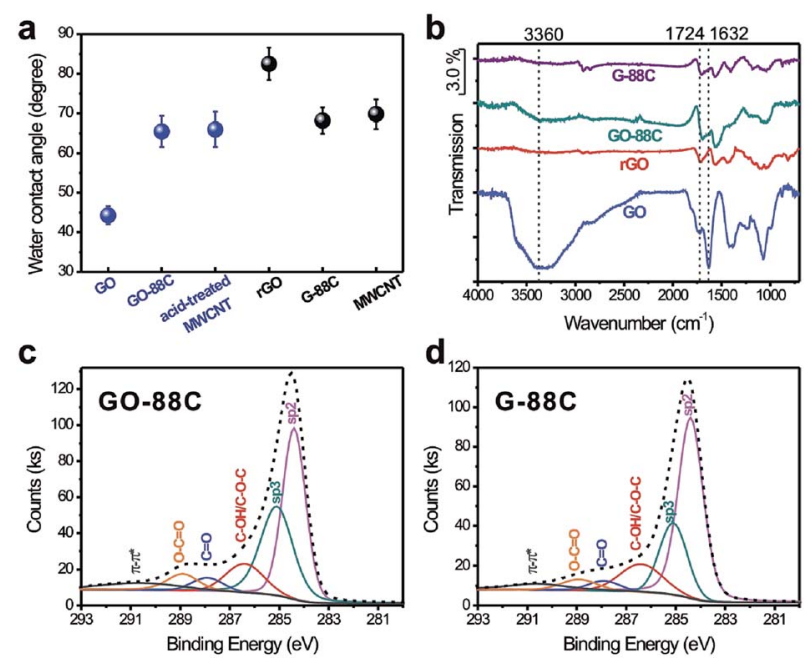

Fig. 5 Water contact angles (a), FTIR spectra (b), and C 1s XPS spectra of the carbon-based layers on PVDF before (c) and after the treatment with $\mathrm{HI}(\mathrm{d})$. The contact angle data were recorded using a $3 \mu \mathrm{L}$ water droplet. 
groups are all significantly weakened but still exist. This clearly demonstrates that the reduction is incomplete. The residual oxygen-containing groups are responsible for this wettable surface of the HI-reduced rGO membrane. XPS measurement was conducted to further examine the surface chemistry composition of the carbon-based layers shown in Fig. 5c and d. The C 1s spectra of the rGO-MWCNT photothermal layers are fitted with six components, i.e., 284.3, 285.1, 286.4, 288.0, 288.9 and $290.6 \mathrm{eV}$, corresponding to $\mathrm{C}=\mathrm{C}$ ( $\mathrm{sp}^{2}$ hybridized), $\mathrm{C}-\mathrm{C}\left(\mathrm{sp}^{3}\right.$ hybridized), $\mathrm{C}-\mathrm{O}$ (epoxy or alkoxy), $\mathrm{C}=\mathrm{O}$ (carbonyl), and $\mathrm{O}=\mathrm{C}-$ $\mathrm{OH}$ (carboxyl) groups, and the $\pi-\pi^{*}$ shake-up satellite structure characteristic of conjugated systems, respectively. ${ }^{39-41}$ Among them, the relative intensities of $\mathrm{C}-\mathrm{O}$ and $\mathrm{C}=\mathrm{O}$ peaks turn weak after the HI reduction treatment. This confirms the decrease of oxygen-containing groups during the reduction process, which leads to the increase of CA. However, the residual oxygencontaining groups contribute to the hydrophilic photothermal layer. And the high surface roughness induces higher hydrophilicity. ${ }^{42}$ With the porous structure, the hydrophilic properties can provide an efficient water supply via the capillary condensation effect. ${ }^{32}$

\subsection{Photothermal evaporation performance of the rGO- MWCNT membranes}

To systematically evaluate the photothermal performance of the carbon-based membranes, the water evaporation rates were recorded by monitoring the real-time weight change under simulated solar irradiation with an intensity of $1 \mathrm{~kW} \mathrm{~m} \mathrm{~m}^{-2}$ equaling to one sun. Fig. 6a schematically exhibits the homemade system for the photothermal evaporation test. The

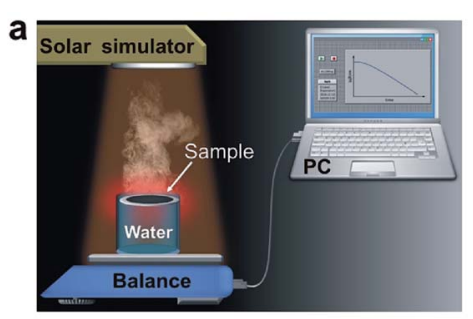

C
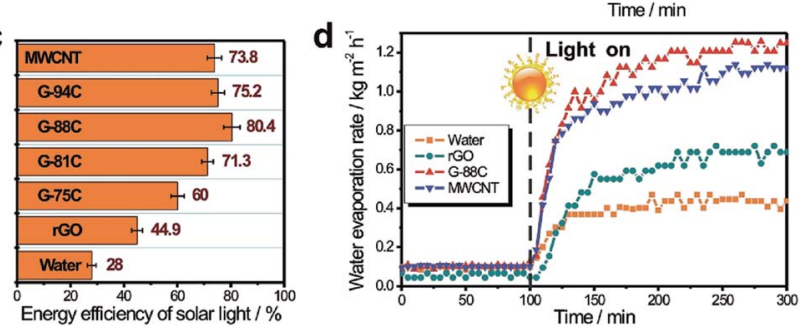

Fig. 6 Schematic illustration of the setup of the solar-driven water evaporation measurement system with the as-prepared carbon-based membrane floating on the surface of water (a). Time course of water evaporation performance under one sun solar irradiation (b) and the corresponding solar thermal conversion efficiency at equilibrium (c). Time course of water evaporation rates without (before $100 \mathrm{~min}$ ) and with solar irradiation (after $100 \mathrm{~min}$ ). Without illumination, the evaporation temperatures are all the same as the ambient temperature, 24 $\pm 0.5^{\circ} \mathrm{C}$, used here (d). tested samples were covered by a polystyrene (PS) foam ring to minimize water evaporation from the gaps between the membrane and the container. As shown by the results in Fig. $6 \mathrm{~b}$ and $\mathrm{S} 8, \dagger$ when the rGO membrane floated on the water, the water evaporation rate increased to $0.68 \mathrm{~kg} \mathrm{~m}^{-2} \mathrm{~h}^{-1}$, about 1.6 times the value obtained without the photothermal membrane (about $0.42 \mathrm{~kg} \mathrm{~m}^{-2} \mathrm{~h}^{-1}$ ). The evaporation can be much faster with the assistance of rGO-MWCNT composite membranes. After the optimization of the MWCNT weight percentage, it is clear that water evaporation rates were significantly increased when MWCNTs were introduced into the rGO photothermal layers. In our present studies, the G-88C membrane (on PVDF) showed the highest evaporation rate of about $1.22 \mathrm{~kg} \mathrm{~m}^{-2} \mathrm{~h}^{-1}$, which is $79 \%$ higher compared to the value obtained for the pure rGO membrane $\left(0.68 \mathrm{~kg} \mathrm{~m}^{-2} \mathrm{~h}^{-1}\right)$. However, with increasing the MWCNT content to $94 \mathrm{wt} \%$, the evaporation rate slows down to $1.14 \mathrm{~kg} \mathrm{~m}^{-2} \mathrm{~h}^{-1}$, which is close to the result observed for the pure MWCNT membrane (around $1.12 \mathrm{~kg} \mathrm{~m}^{-2} \mathrm{~h}^{-1}$ ). These results agree with the light reflectivity measurement results, which also show that sample G-88C possesses the best light absorption capability.

The solar thermal conversion efficiency of solar light $(\eta)$ is another parameter used to assess the photothermal performance for water evaporation and is defined in eqn (2), ${ }^{18}$

$$
\eta=\frac{\left(H_{\mathrm{v}}+H_{\mathrm{s}}\right) \times v_{\mathrm{e}}}{Q}
$$

where $H_{\mathrm{v}}$ is the heat of vaporization $\left(2260 \mathrm{~kJ} \mathrm{~kg}^{-1}\right), H_{\mathrm{s}}$ is the sensible heat, $v_{\mathrm{e}}$ is the water evaporation rate (directly obtained from Fig. $\mathrm{S} 8 \dagger$ ), and $Q$ is the power of simulated solar light for vertical irradiation $\left(1 \mathrm{~kW} \mathrm{~m}^{-2}\right)$. As shown in Fig. $6 \mathrm{c}$, the maximal solar thermal conversion efficiency of the rGOMWCNT membrane (on PVDF) reaches $80.4 \%$, which is significantly higher than the value observed for the pure rGO on the PVDF membrane (44.9\%). It should be noted that this value was achieved in a system without optimizing the heat transfer. This solar thermal conversion efficiency of G-88C can even match the values reported for some elaborate materials even under one sun irradiation (Table S1†). ${ }^{22,32,43-45}$

The variation trend in the evaporation performance of the carbon-based membranes can be discussed with the solar-light absorbance impacted by the surface micro-structure. With the MWCNT composite, the rGO-MWCNT membranes exhibit high surface roughness and higher light absorbances of around 95\%. The efficient light harvesting leads to a higher surface temperature and thereby contributes to faster evaporation.

Moreover, effective water (vapor) transfer in photothermal membranes is another important factor that accelerates the water evaporation. To exclude the influence of PVDF substrates, the evaporation performance of the pristine PVDF membrane was initially tested under irradiation as shown in Fig. S9. $\dagger$ The evaporation rate of the PVDF substrate is about $0.43 \mathrm{~kg} \mathrm{~m}^{-2} \mathrm{~h}^{-1}$, similar to the result of pure water. This result confirms that the hydrophilic surface and porous structure of PVDF ensure sufficient water supply and its influence on water evaporation was negligible. Therefore, the effect of PVDF membrane on the water transport and evaporation process was neglected in the 
following discussions. Many previous literature reports in the field of filtration showed that it was difficult for water molecules to pass through the rGO layer owing to its closely stacked layered structure. ${ }^{29,46}$ As shown in Fig. 6d, the natural evaporation rate with the pure rGO membrane is only $0.06 \mathrm{~kg} \mathrm{~m}^{-2} \mathrm{~h}^{-1}$, which is comparatively lower than the rate obtained for the pure water $\left(0.10 \mathrm{~kg} \mathrm{~m}^{-2} \mathrm{~h}^{-1}\right)$ in the absence of light, while sample $\mathrm{G}-$ 88C and pure MWCNT membranes both show evaporation rates of around $0.10 \mathrm{~kg} \mathrm{~m}^{-2} \mathrm{~h}^{-1}$, similar to the rate of pure water without illumination, indicating that the photothermal layer does not impede the natural water evaporation process. Meanwhile, the equal evaporation rates of G-88C and MWCNT membranes without illumination would illustrate that water transport is not a key factor to explain the better evaporation performance of G-88C than pure MWCNTs. We believe that the loose structure and wettable surface of the rGO-MWCNT layer ensure feasible water transport, while the dense pure rGO membrane will impede the water evaporation due to the lack of water transport channels inside the membrane.

The surface temperature of the photothermal membrane floating on water can also be used to explain the role of water transport in evaporation. The water evaporation rate at different temperatures can be estimated through the empirical formula below (eqn (3)): ${ }^{13}$

$$
v_{\mathrm{c}}=C \theta\left(\frac{p_{\mathrm{sw}}}{p_{\mathrm{a}}-p_{\mathrm{sw}}}-\frac{\varphi p_{\mathrm{sw}}}{p_{\mathrm{a}}-\varphi p_{\mathrm{sw}}}\right)
$$

where $v_{\mathrm{c}}$ is the calculated water evaporation rate $\left(\mathrm{kg} \mathrm{m}^{-2} \mathrm{~h}^{-1}\right)$, $p_{\mathrm{a}}$ and $p_{\mathrm{sw}}$ are the atmospheric pressure and saturation pressure of water vapor at a certain temperature respectively $(\mathrm{kPa}), \varphi$ is the relative humidity in the atmosphere, $\theta$ is a parameter relevant to air velocity above the water surface, and $C$ is an empirical constant with a value of 0.62198 . $\varphi$ remained at around $50 \%$. And $\theta$ was set to 25 for no wind in our test system.

Table 1 presents the surface equilibrium temperatures (on the water) and the theoretical evaporation rates with or without photothermal membranes under one sun irradiation. As the pure water surface temperature reached $34{ }^{\circ} \mathrm{C}$, the evaporation rate calculated from the eqn (3) was $0.43 \mathrm{~kg} \mathrm{~m}^{-2} \mathrm{~h}^{-1}$, in good agreement with the experimental value, while the rGO membrane showed a surface temperature of about $44{ }^{\circ} \mathrm{C}$ at equilibrium, corresponding to $0.80 \mathrm{~kg} \mathrm{~m}^{-2} \mathrm{~h}^{-1}$ of the theoretical evaporation rate. However, this result was significantly higher than the evaporation rate obtained in our experiment $\left(0.68 \mathrm{~kg} \mathrm{~m}^{-2} \mathrm{~h}^{-1}\right)$. The abnormal evaporation rate difference between the calculated and obtained values of rGO is mainly due to its dense layered structure, thereby leading to inferior water transport. In contrast, the G-88C and MWCNT membranes both showed higher experimental evaporation rates, 1.22 and $1.12 \mathrm{~kg} \mathrm{~m}^{-2} \mathrm{~h}^{-1}$, respectively, than the theoretical values calculated ( 0.96 and $0.90 \mathrm{~kg} \mathrm{~m}^{-2} \mathrm{~h}^{-1}$ respectively). The good water transport would be considered as one of the reasons to ensure high-efficiency evaporation.

Among the photothermal samples presented here, G-88C shows the best performance for photothermal evaporation. The improved performance of $\mathrm{G}-88 \mathrm{C}$ could be due to the following two aspects. Firstly, with the suitable MWCNT ratio, the surface of $\mathrm{G}-88 \mathrm{C}$ has the highest roughness, which is beneficial to reduce the diffuse reflection for high light harvesting. Better light absorption results in a higher surface temperature under illumination at the same intensity. Secondly, with an $88 \%$ MWCNT ratio, G-88C has a more loose nano-structure due to its improved thickness, thereby ensuring the better water transfer through the photothermal layer. Based on the above factors, G$88 \mathrm{C}$ is the optimum composition that could efficiently accelerate the evaporation process and achieve a high efficiency of solar thermal conversion.

The solar-driven water evaporation performances of the rGOMWCNT membranes were further measured under different water conditions, including simulated seawater (saline water) and simulated domestic wastewater (water with acid, base and detergent). The average water evaporation rates of $\mathrm{G}-88 \mathrm{C}$ are in the range between 1.19 and $1.31 \mathrm{~kg} \mathrm{~m}^{-2} \mathrm{~h}^{-1}$ (Fig. 7). After a 24 hour measurement, the composite photothermal layer was intact and showed stable evaporation performance. We believe that the evaporation rate can be further advanced by thermal management with a thermal barrier layer, just as reported in our previous work. ${ }^{13}$ Such results demonstrate the potential practical application value of our rGO-MWCNT membranes in the fields of desalination and wastewater treatment.

Table 1 The theoretical $\left(v_{c}\right)$ and experimental $\left(v_{\mathrm{e}}\right)$ evaporation rates with different surface temperatures

\begin{tabular}{|c|c|c|c|c|}
\hline $47^{\circ} \mathrm{C}$ & & & & \\
\hline Surface temperature $\left[{ }^{\circ} \mathrm{C}\right]$ & 34 & 44 & 47 & 46 \\
\hline$p_{\mathrm{sw}}[\mathrm{kPa}]$ & 5.32 & 9.11 & 10.62 & 10.09 \\
\hline$v_{\mathrm{c}}\left[\mathrm{kg} \mathrm{m}^{-2} \mathrm{~h}^{-1}\right]$ & 0.43 & 0.80 & 0.96 & 0.90 \\
\hline$v_{\mathrm{e}}\left[\mathrm{kg} \mathrm{m}^{-2} \mathrm{~h}^{-1}\right]$ & 0.42 & 0.68 & 1.22 & 1.12 \\
\hline
\end{tabular}




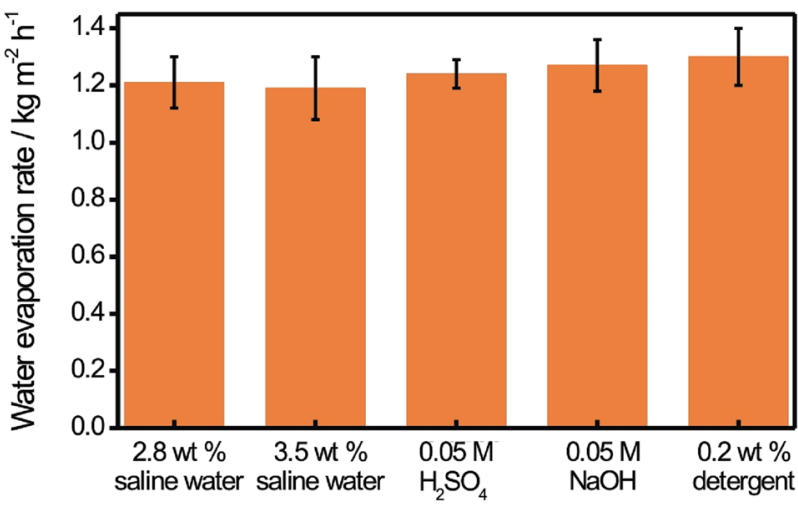

Fig. 7 The average water evaporation rates under different water conditions for over $100 \mathrm{~min}$.

\section{Conclusions}

In conclusion, we have developed a facile nanocomposite strategy to rationally design photothermal membranes using $2 \mathrm{D}$ rGO and 1D MWCNTs for highly efficient solar-driven evaporation. Doping of MWCNTs into rGO-MWCNT photothermal layers results in a controllable surface micro-structure, loose structure and wettable surface, which can improve solar light harvesting and water transport simultaneously for a high evaporation rate under one sun $\left(1 \mathrm{~kW} \mathrm{~m}^{-2}\right)$. The solar thermal conversion efficiency of the optimized rGO-MWCNT photothermal layer on PVDF reaches a maximum of $80.4 \%$, which is significantly higher than the results of pure rGO and MWCNTs. This composite photothermal layer can be deposited on various substrates via a facile and general strategy, which is beneficial to meet different application conditions in the fields of desalination and wastewater treatment. In summary, this work exhibits a rational design concept of a structural composite to enhance the solar-driven evaporation performance for high practical application value in water purification.

\section{Conflicts of interest}

There are no conflicts to declare.

\section{Acknowledgements}

This work was funded by the National Natural Science Foundation of China (21701181), CPSF-CAS Joint Foundation for Excellent Postdoctoral Fellows (2016LH00035), China Postdoctoral Science Foundation (Y63308190V), Shandong Provincial Natural Science Foundation, China (ZR2017BB051), Shandong Provincial Key R\&D Project (2016CYJS07A02) and Qingdao Public Health and Science and Technology Plan (17-33-82-nsh).

\section{Notes and references}

1 T. Oki and S. Kanae, Science, 2006, 313, 1068-1072.

2 Q. Schiermeier, Nature, 2014, 505, 10-11.
3 R. Y. Li, L. B. Zhang and P. Wang, Nanoscale, 2015, 7, 1716717194.

4 X. Gao, H. Y. Ren, J. Y. Zhou, R. Du, C. Yin, R. Liu, H. L. Peng, L. M. Tong, Z. F. Liu and J. Zhang, Chem. Mater., 2017, 29, 5777-5781.

5 L. Zhou, Y. Tan, J. Wang, W. Xu, Y. Yuan, W. Cai, S. Zhu and J. Zhu, Nat. Photonics, 2016, 10, 393-398.

6 L. B. Zhang, B. Tang, J. B. Wu, R. Y. Li and P. Wang, Adv. Mater., 2015, 27, 4889-4894.

7 Y. Liu, J. W. Lou, M. T. Ni, C. Y. Song, J. B. Wu, N. P. Dasgupta, P. Tao, W. Shang and T. Deng, ACS Appl. Mater. Interfaces, 2016, 8, 772-779.

8 O. Neumann, A. S. Urban, J. Day, S. Lal, P. Nordlander and N. J. Halas, ACS Nano, 2013, 7, 42-49.

9 O. Neumann, A. D. Neumann, E. Silva, C. Ayala-Orozco, S. Tian, P. Nordlander and N. J. Halas, Nano Lett., 2015, 15, 7880-7885.

10 M. Calvin, Science, 1974, 184, 375-381.

11 P. V. Kamat, J. Phys. Chem. C, 2007, 111, 2834-2860.

12 Y. C. Wang, L. B. Zhang and P. Wang, ACS Sustainable Chem. Eng., 2016, 4, 1223-1230.

13 L. Shi, Y. Wang, L. Zhang and P. Wang, J. Mater. Chem. A, 2017, 5, 16212-16219.

14 W. Shang and T. Deng, Nat. Energy, 2016, 1, 16133.

15 H. Wang, L. Miao and S. Tanemura, Solar RRL, 2017, 1, 1600023.

16 Z. P. Liu, Z. J. Yang, X. C. Huang, C. Y. Xuan, J. H. Xie, H. D. Fu, Q. X. Wu, J. M. Zhang, X. C. Zhou and Y. Z. Liu, J. Mater. Chem. A, 2017, 5, 20044-20052.

17 Y. Zeng, J. F. Yao, B. A. Horri, K. Wang, Y. Z. Wu, D. Li and H. T. Wang, Energy Environ. Sci., 2011, 4, 4074-4078.

18 H. Ghasemi, G. Ni, A. M. Marconnet, J. Loomis, S. Yerci, N. Miljkovic and G. Chen, Nat. Commun., 2014, 5, 4449.

19 Z. Liu, H. Song, D. Ji, C. Li, A. Cheney, Y. Liu, N. Zhang, X. Zeng, B. Chen, J. Gao, Y. Li, X. Liu, D. Aga, S. Jiang, Z. Yu and Q. Gan, Global Challenges, 2017, 1, 1600003.

20 Y. M. Liu, J. W. Chen, D. W. Guo, M. Y. Cao and L. Jiang, ACS Appl. Mater. Interfaces, 2015, 7, 13645-13652.

21 L. Zhou, S. D. Zhuang, C. Y. He, Y. L. Tan, Z. L. Wang and J. Zhu, Nano Energy, 2017, 32, 195-200.

22 R. Li, L. Zhang, L. Shi and P. Wang, ACS Nano, 2017, 11, 3752-3759.

23 G. Wang, Y. Fu, X. F. Ma, W. B. Pi, D. W. Liu and X. B. Wang, Carbon, 2017, 114, 117-124.

24 X. Q. Wang, G. Ou, N. Wang and H. We, ACS Appl. Mater. Interfaces, 2016, 8, 9194-9199.

25 H. Ren, M. Tang, B. Guan, K. Wang, J. Yang, F. Wang, M. Wang, J. Shan, Z. Chen, D. Wei, H. Peng and Z. Liu, Adv. Mater., 2017, 29, 1702590.

26 B. Tang, L. B. Zhang, R. Y. Li, J. B. Wu, M. N. Hedhili and P. Wang, Nanoscale, 2016, 8, 1108-1116.

27 K. Yang, X. Zhu and B. Chen, J. Mater. Chem. A, 2017, 5, 20316-20326.

28 P. Zhang, J. Li, L. Lv, Y. Zhao and L. Qu, ACS Nano, 2017, 11, 5087-5093.

29 Y. Su, V. G. Kravets, S. L. Wong, J. Waters, A. K. Geim and R. R. Nair, Nat. Commun., 2014, 5, 4843. 
30 H. Y. Liu, H. T. Wang and X. W. Zhang, Adv. Mater., 2015, 27, 249-254.

31 J. Yang, X. F. Li, S. Han, Y. T. Zhang, P. Min, N. Koratkar and Z. Z. Yu, J. Mater. Chem. A, 2016, 4, 18067-18074.

32 Y. Ito, Y. Tanabe, J. H. Han, T. Fujita, K. Tanigaki and M. W. Chen, Adv. Mater., 2015, 27, 4302-4307.

33 H. Hu, Z. B. Zhao, R. Zhang, Y. Z. Bin and J. S. Qiu, J. Mater. Chem. A, 2014, 2, 3756-3760.

34 H. Hu, Z. B. Zhao, W. B. Wan, Y. Gogotsi and J. S. Qiu, Adv. Mater., 2013, 25, 2219-2223.

35 D. C. Marcano, D. V. Kosynkin, J. M. Berlin, A. Sinitskii, Z. Z. Sun, A. Slesarev, L. B. Alemany, W. Lu and J. M. Tour, ACS Nano, 2010, 4, 4806-4814.

36 Y. C. Wang, S. Y. Tao and Y. L. An, J. Mater. Chem. A, 2013, 1, 1701-1708.

37 Y. C. Wang, S. Y. Tao and Y. L. An, Microporous Mesoporous Mater., 2012, 163, 249-258.

38 S. Y. Tao, Y. C. Wang and Y. L. An, J. Mater. Chem., 2011, 21, 11901-11907.
39 J. L. Zhang, H. J. Yang, G. X. Shen, P. Cheng, J. Y. Zhang and S. W. Guo, Chem. Commun., 2010, 46, 1112-1114.

40 S. Stankovich, D. A. Dikin, R. D. Piner, K. A. Kohlhaas, A. Kleinhammes, Y. Jia, Y. Wu, S. T. Nguyen and R. S. Ruoff, Carbon, 2007, 45, 1558-1565.

41 K. Haubner, J. Murawski, P. Olk, L. M. Eng, C. Ziegler, B. Adolphi and E. Jaehne, ChemPhysChem, 2010, 11, 21312139.

42 L. Feng, S. H. Li, Y. S. Li, H. J. Li, L. J. Zhang, J. Zhai, Y. L. Song, B. Q. Liu, L. Jiang and D. B. Zhu, Adv. Mater., 2002, 14, 1857-1860.

$43 \mathrm{X} . \mathrm{Hu}, \mathrm{W} . \mathrm{Xu}, \mathrm{L}$. Zhou, Y. Tan, Y. Wang, S. Zhu and J. Zhu, Adv. Mater., 2017, 29, 1604031.

44 Y. Y. Chen, X. J. Quan, Z. Y. Wang, C. S. Lee, Z. Z. Wang, P. Tao, C. Y. Song, J. B. Wu, W. Shang and T. Deng, J. Mater. Chem. A, 2016, 4, 17503-17511.

45 Y. Fu, G. Wang, T. Mei, J. H. Li, J. Y. Wang and X. B. Wang, ACS Sustainable Chem. Eng., 2017, 5, 4665-4671.

46 Y. Han, Z. Xu and C. Gao, Adv. Funct. Mater., 2013, 23, 36933700 . 